\title{
Active Surveillance Of Endo-Tracheal Aspirates From Mechanically Ventilated Patients In Intensive Care Unit At A Tertiary Care Center
}

\author{
Priya Santharam ${ }^{1}$, Sudha $K^{2}$, Shanmugavadivoo $N^{3}$, Usha $B^{4}$, Padmavathi B $K^{5}$ \\ ${ }^{1,2}$ Assistant Professor, Department of Microbiology Annapoorna Medical College \& Hospital, Salem. \\ ${ }^{3}$ Associate Professor, Department of Microbiology, Annapoorna Medical College \& Hospital, Salem. \\ ${ }^{4}$ Prof \& HOD, Department of Microbiology, Annapoorna Medical College \& Hospital, Salem. \\ ${ }^{5}$ Prof \& HOD, Department of Microbiology, Kerala Medical College, Mangode, Palakkad, Kerala
}

\begin{abstract}
Introduction: Critically ill Intensive care unit (ICU) patients are most vulnerable for developing respiratory and urinary tract infections especially who are mechanically ventilated due to complex interplay between the endotracheal tube, host immunity and virulence of invading bacteria. The aim and objective of the study was to analyze the aerobic bacteria and their antibiotic sensitivity pattern isolated from endotracheal aspirates of ventilated patients in ICU.

Materials and Methods: The prospective study was done with the endotracheal samples received in the Microbiology Laboratory in a tertiary care hospital in south India, over a period of one year. Samples were collected under sterile conditions from medical and surgical ICU patients who were ventilated for at least $48 \mathrm{hrs}$. The samples included endotracheal secretion and endotracheal tube tip which was processed as per microbiological standards.
\end{abstract}

Results: A total of 41 samples from 41 patients were collected during the study period of one year. The two major reasons for admission were organophosphate poisoning and RTA with head injury. There was predominance of male patients with M: F ratio of 3:1 respectively. The organisms commonly isolated were Acinetobacter, Pseudomonas, and Staphylococcus aureus. Out of 41 isolates 21 were multi-drug resistant which implies that $50 \%$ of isolates are multidrug resistant.

Conclusion: Regular periodic surveillance of microbial profile and susceptibility patterns of ventilated patients in ICU should be done to detect emerging resistant bacterial strains. To combat the high antimicrobial resistance, every center should adopt a strict hospital infection control policy which includes judicious use of antimicrobial agents, use of rational antibiotic therapy, education programs and hand hygiene.

Keywords: Antibiotic policies, endotracheal aspirates, hand hygiene, multi-drug resistant, ventilator

\section{INTRODUCTION:}

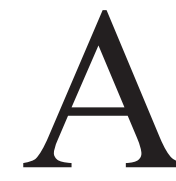

$\mathrm{n}$ intensive care unit (ICU) is a special department of a hospital that cater to patients with the most severe and lifethreatening illnesses and injuries; that require constant, close monitoring and support from specialized equipment and medication in order to maintain normal vital functions of the patient. ${ }^{1}$ This increases the risk of the ICU patient five to seven fold with an increase in mortality due to nosocomial infection. Intensive care units contribute $20 \%$ to $25 \%$ of all nosocomial infections in a hospital. ${ }^{2,3}$ Critically ill Intensive care unit (ICU) patients are most vulnerable for developing 
respiratory and urinary tract infections as compared to those patients admitted in ward.

Respiratory infections in critically ill patients are associated with high morbidity and mortality. ${ }^{4}$ Rapid diagnosis and initiation of appropriate antibiotic therapy is essential for better outcomes. Patients who are intubated and mechanically ventilated are further at risk of acquiring respiratory infections due to complex interplay between the endotracheal tube, host immunity and virulence of invading bacteria. The etiological agents may vary according to the population of patients in an ICU, duration of hospital stay, preexisting illness and prior antimicrobial therapy. ${ }^{5}$ Common causative agents are Pseudomonas species, Acinetobacter species, Staphylococcus aureus, and Enterobacteriaceae including the endogenous bacteria ${ }^{6}$. The common problem however as noted in other studies is that bacterial pathogens from tracheal aspirates tend to be a multidrug-resistant. ${ }^{7}$ There is much well documented evidence that hospital personnel and environment are the microbial source and prolonged hospital stay and overuse of antimicrobial agents has led to multidrug resistance of these microbes. ${ }^{8}$

In the hospital, endotracheal aspiration for the diagnosis of nosocomial infection is common in patients admitted in the ICU. ${ }^{9}$ Therefore updated knowledge of local epidemiological and susceptibility profile is recommended for guiding the clinicians regarding empirical choice of antibiotics and has become mandatory along with adequate clinical diagnosis and bacterial confirmation. ${ }^{10}$ Hence the aim and objective of the study was to analyze the spectrum of aerobic bacteria and their antibiotic sensitivity pattern isolated from endotracheal aspirates of ventilated patients in Intensive Care Units.

\section{AIMS AND OBJECTIVES:}

To analyze the aerobic bacteria and their antibiotic sensitivity pattern isolated from endotracheal aspirates of ventilated patients in ICU.

\section{MATERIALS AND METHODS:}

The prospective study was carried out with the samples of endotracheal aspirate and endotracheal tube tip received in the Microbiology Laboratory over a period of one year (Jan 2013 - Dec 2013). Samples were collected under sterile conditions from patients admitted in both medical and surgical intensive care unit who were ventilated for at least 48 hrs. In patients who were ventilated outside, samples were taken on day of admission. The samples included endotracheal secretion and endotracheal tube tip.

\section{Collection of Specimen:}

\section{Endotracheal Aspirate:}

Sampling was done by introducing a catheter aseptically through the endotracheal tube and secretions aspirated into a sterile syringe. ${ }^{11}$

\section{Endo-tracheal tube tip:}

The endo-tracheal tube tip was cut aseptically into a sterile container and sent to the Microbiology laboratory.

\section{Processing of Specimen:}

\section{Endotracheal Aspirate:}

Both $10 \mu 1$ of the specimen and $1 \mu 1$ of the specimen was inoculated in blood agar and MacConkey agar. $^{12}$

\section{Endotracheal tube tip:}

The lumen of endotracheal tube tip was rinsed with $0.5 \mathrm{ml}$ of sterile normal saline. $10 \mu 1$ of the fluid was inoculated on blood agar and MacConkey agar. 
A gram stain of the endotracheal secretions / endotracheal tube tip fluid was done to assess the number of pus cells and the presence of bacteria. A semi quantitative method was followed and plates were incubated overnight at $37^{\circ} \mathrm{C} .{ }^{13} \mathrm{~A}$ significant growth of $>10^{5} \mathrm{CFU} / \mathrm{ml}$ was taken for identification. The organisms isolated were identified based on colony characteristics on Blood agar and MacConkey agar, Gram's stain, Biochemical reactions by using standard microbiological techniques. Isolates identified as commensals or contaminants were excluded from further process. A Kirby-Bauer method was used to test the susceptibility of organisms to various antibiotics.

\section{Antibiotics used:}

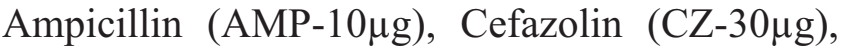
Cefoxitin (CX-30 $\mu \mathrm{g})$, Vancomycin (VA-30 $\mu \mathrm{g})$,

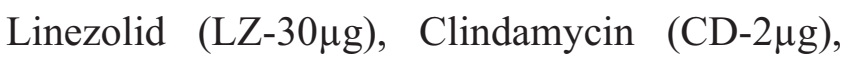
Erythromycin $(\mathrm{E}-15 \mu \mathrm{g})$, Piperacillin (PI-100 $\mu \mathrm{g})$, Ceftazidime (CAZ-30 $\mu \mathrm{g})$, Ceftazidime/Clavulanic acid (CAC-30 $\mu \mathrm{g} / 10 \mu \mathrm{g})$, Cefeperazone (CPZ$30 \mu \mathrm{g})$, Cefotaxime (CTX-30 $\mu \mathrm{g})$,Tobramycin

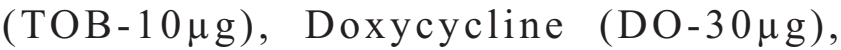
Piperacillin/Tazobactum (PIT-100 $\mu \mathrm{g} / 10 \mu \mathrm{g}$ ),

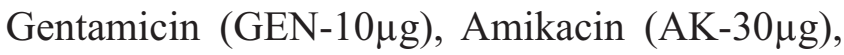
Azteronam (AT-30 $\mu \mathrm{g}$ ), Ciprofloxacin (CIP-5 $\mu \mathrm{g}$ ), Ofloxacin (OF-5 $\mu \mathrm{g}$ ), Imipenem (IMP-10 $\mu \mathrm{g}$ ), Colistin (CL-10 $\mu \mathrm{g})$, Polymyxin B (PB-300U). Zone diameter was measured and interpreted as per the Clinical and Laboratory Standards Institute (CLSI) guidelines.

\section{RESULTS:}

A total of 41 samples from 41 patients were collected during the study period of one year. The major reason for admission was organophosphate poisoning and second major reason being RTA with head injury. There was male predominance with a male to female ratio of 3:1 respectively. Most of the patients fell into the age group of 20-30 years. Endo -tracheal tube tip was the majority sample received and was processed as per CLSI guidelines. There was growth in 37 specimens and 4 specimens were sterile. The organisms isolated were Acinetobacter spp (14), Pseudomonas spp (9), Staphylococcus aureus (8), Klebsiella pneumoniae (4), Nonfermenting GNB - NFGNB (4), Escherichia coli (1) and Citrobacter spp (1) (Table 1). Susceptibility pattern of isolated organisms is shown in (Fig 1 4). Among the eight Staphylococcus aureus all the strains were Methicillin resistant (Cefoxitin resistant). Out of fourteen Acinetobacter spp, nine of them were multi-drug resistant. Two multi-drug resistant strain out of nine Pseudomonas isolates. There were six multi-drug resistant strains out of eight Klebsiella pneumoniae. Escherichia coli, Citrobacter spp and NFGNB were all multi-drug resistant and were only susceptible to Imipenem.

Table 1. Distribution of Bacterial Isolates from endo-tracheal tube aspirates

\begin{tabular}{|l|c|}
\hline Bacterial Isolates & No. of isolates \\
\hline Acinetobacter spp & 14 \\
\hline Pseudomonas spp & 9 \\
\hline Staphylococcus aureus & 8 \\
\hline Klebsiella pneumoniae & 4 \\
\hline NFGNB & 4 \\
\hline Escherichia coli & 1 \\
\hline Citrobacter spp & 1 \\
\hline \multicolumn{1}{|c|}{ Total } & $\mathbf{4 1}$ \\
\hline
\end{tabular}


Figure 1. Sensitivity pattern of Staphylococcus aureus

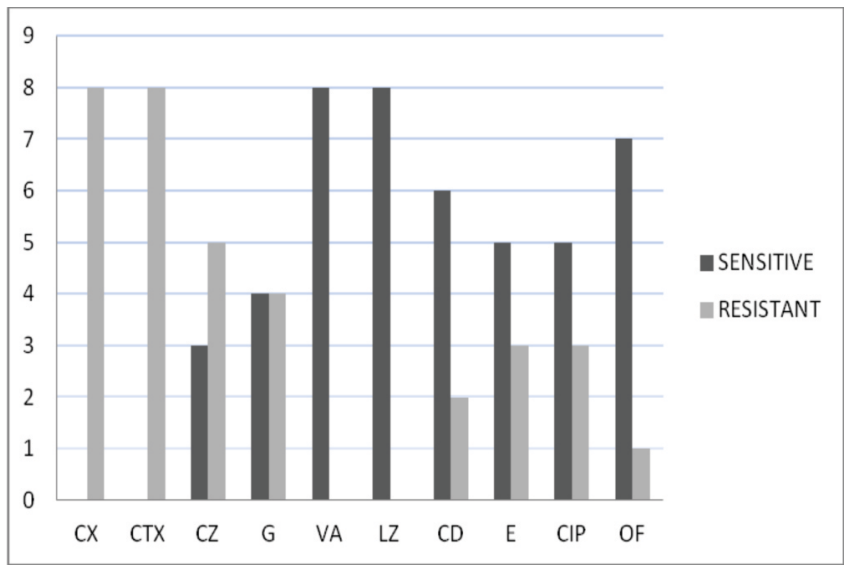

Figure 2. Sensitivity pattern of Acinetobacter spp

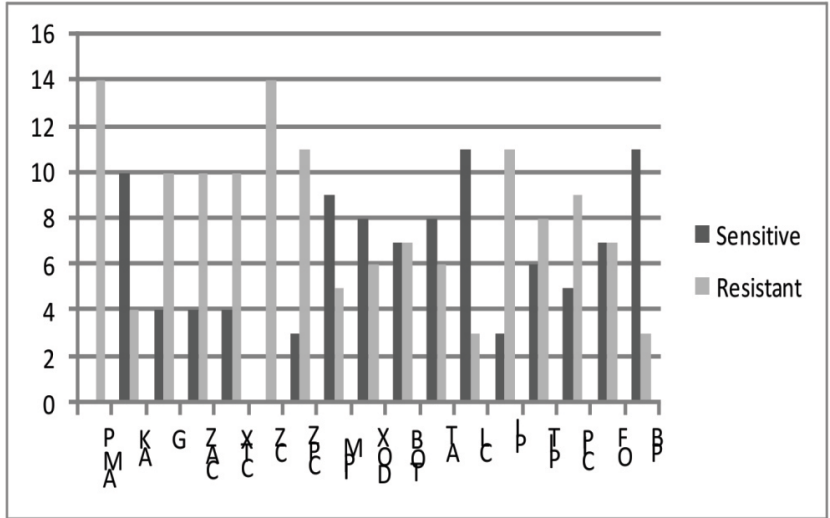

Figure 3. Sensitivity pattern of Pseudomonas spp

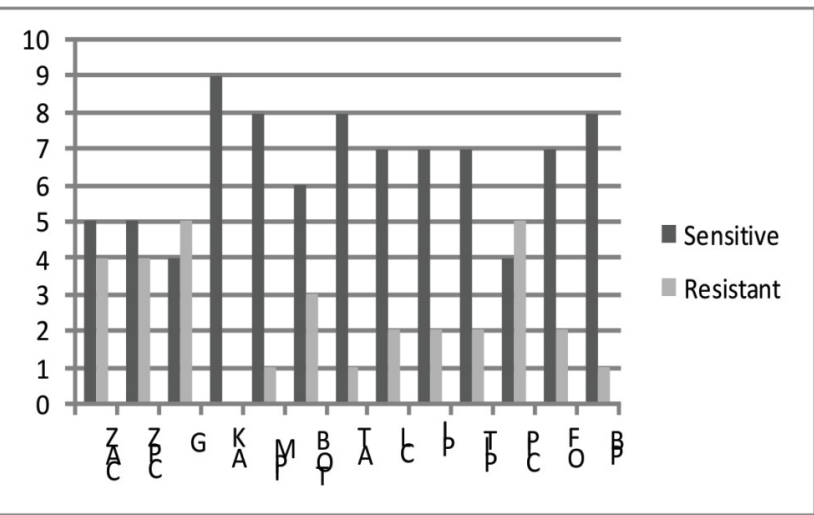

Figure 4. Sensitivity pattern of Klebsiella pneumoniae

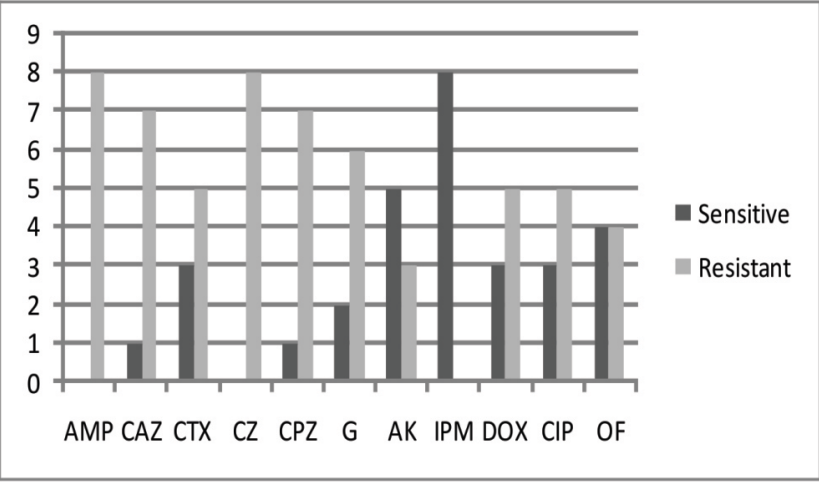

\section{DISCUSSION:}

Endotracheal intubation and mechanical ventilation are life-saving procedures done on emergency or elective basis to prevent or combat respiratory failure. Many clinical conditions warrant need for ventilator support like, life threatening infections, sepsis and acute respiratory distress syndromes, neurological dysfunctions due to poisoning, drug toxicity, cerebrovascular accidents, trauma and others. On one hand while mechanical ventilation helps to prevent deaths due to respiratory failure on the other hand it poses great threat, by host immune response and infectious organisms that leads to life threatening lung infections. ${ }^{5}$ The mechanically ventilated and tracheotomised patients are colonized with bacteria of either endogenous or exogenous origin which might end up in VAT or VAP. ${ }^{8}$ Health care associated infections (HCAI) continue to be a major cause of patient morbidity and mortality in ICU.

In our study the most frequent clinical condition needing mechanical ventilation was poisoning and trauma (Head injury) was the second common condition. In a study by Arindam dey et $\mathrm{al}^{14}$, postoperative condition was the most frequent clinical 
Priya Santharam, et.al : Active Surveillance of endo-tracheal aspirates from mechanically ventilated

condition needing mechanical ventilation and COPD patients were the most frequently ventilated patients in a study by Ramakrishna pai et al. ${ }^{15} \mathrm{~A}$ study by Shanmugavadivoo et $\mathrm{al}^{8}$ showed results similar to our study. Our study showed a predominance of male ventilated patients than female in the ratio of $3: 1$ respectively which is correlated with the study conducted by Saha et al. ${ }^{6}$

In the present study, organisms isolated from endotracheal aspirates were Acinetobacter spp (14), Pseudomonas spp (9), Staphylococcus aureus (8), Klebsiella pneumoniae (4), Non-fermenting GNB (4), Escherichia coli (1) and Citrobacter spp (1). The predominant organism was Acinetobacter followed by Pseudomonas, Staphylococcus aureus and Klebsiella pneumoniae. Our result has been correlated with Shanmugavadivoo et $\mathrm{al}^{8}$ that Acinetobacter has overtaken the pathogenic role in ventilated patients. A study by Tihana MT et $\mathrm{al}^{16}$ showed Pseudomonas as a predominant organism in endotracheal aspirates. All the S.aureus strains were Methicillin resistant Staphylococcus aureus which is a most common causative agent of nosocomial infection.

In this study, microbial profile of endo-tracheal aspirates in ventilated patients showed an increase in number of Acinetobacter spp, of which nine of them were multi-drug resistant. Colistin is considered the treatment option for critically ill patients in the ICU with infections caused by multidrug resistant Acinetobacter spp, owing to its favorable properties of rapid bacterial killing and an associated slow development of resistance ${ }^{17}$. In our study three Acinetobacter showed Colistin resistance. Bassetti $\mathrm{M}$ et $\mathrm{al}^{18}$ showed that a combination of Colistin and Rifampicin appears to be an effective and safe therapy for severe infections due to multidrug - resistant Acinetobacter spp. ${ }^{19}$ In our study multi-drug resistant Acinetobacter (9), Pseudomonas (2), Klebsiella pneumonia (6), Non fermenting Gram Negative bacilli (2), E.coli (1) and Citrobacter (1) has been isolated. Out of 41 isolates 21 are multidrug resistant which implies that $50 \%$ of isolates are multidrug resistant. The major reasons for multidrug resistance are ventilators, prolonged hospital stay, irrational usage of antibiotics. There are many studies that has been shown similar results. $^{1,2,5,20,21}$

\section{CONCLUSION:}

In our study we found that Gram negative bacteria especially Acinetobacter is the most common etiological agent in tracheotomised patients. Most of the organisms were multi-drug resistant. This study has set up new research goal for surveillance of microbial profile and susceptibility patterns of ventilated patients in ICU. This should be conducted regularly in order to detect potential alterations, especially emerging resistant bacterial strains. This study can only be effective if there is a standard antibiotic policy in the hospital which is reviewed at periodic intervals by a hospital infection control committee. So, to combat the high antimicrobial resistance, every center should have a strict hospital infection control policy which includes measures like judicious use of antimicrobial agents, use of rational antibiotic therapy, education programs and hand hygiene.

\section{ACKNOWLEDGEMENT:}

My sincere thanks to the Medical \& Nursing staff of ICU, for their continued support in procurement of the data and the microbiology laboratory technicians, who were of immense help in the 
processing of samples for the study. A note of thanks to Mr Vignesh Kanna, for his help in the compilation of this paper.

\section{REFERENCES:}

1. Lamya H, Mostofa K, Zakaria A. Isolation, identification and antimicrobial sensitivity pattern of bacterial isolates from tracheal aspirate of ICU patients of central Dhaka, Bangladesh. Intl J Res App Nat Soc Sci 2013; 1(7):11-16.

2. Bhasie M, Ostwal K, Shah P, Waghmare P, Ingole $\mathrm{K}$, Powar $\mathrm{K}$. Isolation, identification and antimicrobial sensitivity pattern of bacterial isolates from tracheal aspirate of ICU patients at tertiary care centre in Solapur. Intl J Sci Res 2015; 4(11):504-6.

3. World Health Organization. Prevention of hospitalized-acquired infections: a practical guide. World Health Organization, Geneva. Switzerland 2002

4. Shalini S, Kranthi K, Gopalkrishna BK. The microbiological profile of nosocomial infections in the intensive care unit. J Clin and Diag Res. 2010; (4):3109-12

5. Anusha N, Madhu KP, Arun BJ, Vidyasagar B. Microbiological profile and sensitivity pattern of endotracheal secretions in mechanically ventilated patients in ICU. J Evi Med Health 2014; 1(9):1177-84

6. Ashis KS, Suman N, Payodhi D. Prevalence of bacterial isolates in endotracheal tube according to culture and sensitivity in patients of intensive care unit of a tertiary medical college and hospital, Kolkata, West Bengal. Intl J Cont Med Res 2016; 3(6):1775-81.
7. Alain CJ, Dominador GM, Gemma BR, Michael AD, Christine TG. Review on the antimicrobial resistance of pathogens from tracheal and endotracheal aspirates of Patients with clinical manifestations of Pneumonia in Bacolod city in 2013.Intl J Bact 2015; 5(8): 1-5.

8. Shanmugavadivoo N, P Santharam, Sudha K, Kalaiselvi G, Padmavathi BK, Usha B, et al. Dynamic bacterial profile of endotracheal aspirates and its sensitivity pattern-A Cause of Concern. Int J Cur Res Rev 2014; 6(10):112-19.

9. Joseph NM, Sistla S, Dutta TK, Badhe AS, Parija SC. Ventilator-associated pneumonia: role of colonizers and value of routine endotracheal aspirate cultures. Int J Infect Dis. 2010; 14(8):723-9

10. Joao M, Ederlon R. Epidemiological and microbiological analysis of ventilatorassociated pneumonia patients in a public teaching hospital. Brazl J infect dis 2007; 11(5):482-8.

11. Koneman EW, Allen SD, Janda WM, Schreckenberger PC, Winn, Jr. WC. Color atlas and textbook of diagnostic microbiology 5th ed. (Lippincot, Philadelphia, 1997), Pp27

12. Shin YM, et al. Usefulness of quantitative endotracheal aspirate cultures in intensive care unit patients with suspected Pneumonia. J Korean Med Sci 2011; 26:865-9.

13. Albert S, Kirchner J, Thomas H, Behne M, Schur J, Brade V et al. Role of quantitative cultures and microscopic examinations of 
Priya Santharam, et.al : Active Surveillance of endo-tracheal aspirates from mechanically ventilated

endotracheal aspirates in the diagnosis of pulmonary infections in ventilated patients. $\mathrm{J}$ Hosp Infect 1997; 37:25-37

14. Arindam Dey, Indira Bairy. Incidence of multidrug-resistant organisms causing ventilator-associated pneumonia in a tertiary care hospital: A nine months prospective study. Ann Thorac Med 2007; 2(2):52-7.

15. Ramakrishna P, Rekha B. Characterization of aerobic bacteria isolated from endotracheal aspirate in adult patients suspected ventilator associated pneumonia in a tertiary care center in Mangalore. Saudi J Anaesth 2012; 6(2):115-9

16. Tihana MT, Ana GG, Branka DC, Bozena G, Mladen S, Mladen P. Microbial profile and antibiotic susceptibility patterns of pathogens causing ventilator associated pneumonia at Intensive Care Unit, Sestre Milososrdnice university hospital center, Zagreb, Croaoatia. Acta Clin Croat 2015; 54:127-35.

17. Kallel H., Bahloul M, Hergafi L, Akrout M, Ketata W, Chelly $\mathrm{H}$, et al. Colistin as a salvage therapy for nosocomial infections caused by multidrug-resistant bacteria in the ICU. Int J Antimicrob Agents. 2006 Oct;28(4):366-9
18. Bassetti M, Repetto E, Righi E, Boni S, Diverio M, Molinari MP, et al. Colistin and Rifampicin in the treatment of multidrugresistant Acinetobacter baumanni infections. J.Antimicrobiol Chemother 2008; 61(2):417-20.

19. Urmi Jethwani. Antibiotic sensitivity pattern of gram negative bacilli isolated from the lower respiratory tract of ventilated patients in the Intensive Care Unit. Indian Medical Gazette 2014; 180-4.

20. Azar DK, Najmeh P, Effat AM, Alireza M, Fariba A. The Prevalence of bacteria isolated from endotracheal tubes of patients in Golestan hospital, Ahvaz, Iran, and determination of their antibiotic susceptibility patterns. Jundishapur J Microbiol. 2013; 6(1):67-71.

21. George P, Sequiera A. Antimicrobial sensitivity pattern among organisms which were isolated from the endotracheal aspirates of patients with ventilator associated pneumonia. J Clin Diag Res. 2010 December;(4):3397-3401

Received on 07/11/2017 Revised on 07/12/2017 Accepted on 09/12/2017 